\title{
Is the Timed Up and Go test a useful predictor of risk of falls in community dwelling older adults: a systematic review and meta- analysis
}

\author{
Emma Barry ${ }^{\dagger}$, Rose Galvin ${ }^{*}{ }^{\dagger}$ Claire Keogh, Frances Horgan and Tom Fahey
}

\begin{abstract}
Background: The Timed Up and Go test (TUG) is a commonly used screening tool to assist clinicians to identify patients at risk of falling. The purpose of this systematic review and meta-analysis is to determine the overall predictive value of the TUG in community-dwelling older adults.

Methods: A literature search was performed to identify all studies that validated the TUG test. The methodological quality of the selected studies was assessed using the QUADAS-2 tool, a validated tool for the quality assessment of diagnostic accuracy studies. A TUG score of $\geq 13.5$ seconds was used to identify individuals at higher risk of falling. All included studies were combined using a bivariate random effects model to generate pooled estimates of sensitivity and specificity at $\geq 13.5$ seconds. Heterogeneity was assessed using the variance of logit transformed sensitivity and specificity.
\end{abstract}

Results: Twenty-five studies were included in the systematic review and 10 studies were included in meta-analysis. The TUG test was found to be more useful at ruling in rather than ruling out falls in individuals classified as high risk (>13.5 sec), with a higher pooled specificity $(0.74,95 \% \mathrm{Cl} 0.52-0.88)$ than sensitivity $(0.31,95 \% \mathrm{Cl} 0.13-0.57)$. Logistic regression analysis indicated that the TUG score is not a significant predictor of falls $(\mathrm{OR}=1.01,95 \% \mathrm{Cl} 1.00-1.02, \mathrm{p}=0.05)$.

Conclusion: The Timed Up and Go test has limited ability to predict falls in community dwelling elderly and should not be used in isolation to identify individuals at high risk of falls in this setting.

\section{Background}

Falls are a leading cause of injury and activity limitation in older adults and the adverse effects associated with falling result in significant personal, social and economic burden. Approximately $30 \%$ of community dwelling people aged 65 years and over will fall each year [1]. Falls account for $40 \%$ of all injury deaths and lead to $20-30 \%$ of mild to severe injuries ranging from soft tissue injuries to fractures in the elderly [2]. The causes of falling are multi-factorial and include extrinsic (environment-related), intrinsic (person-related) and behavioural (activity-related) factors. Gait instability has been identified as a relatively consistent risk factor for falls and the majority of screening programmes to identify those at risk of falls comprise an

\footnotetext{
* Correspondence: rosegalvin@rcsi.ie

${ }^{\dagger}$ Equal contributors

HRB Centre for Primary Care research, Department of General Practice, Royal College of Surgeons in Ireland, 123 St. Stephens Green, Dublin 2, Republic of Ireland
}

\section{Biomed Central}

(c) 2014 Barry et al.; licensee BioMed Central Ltd. This is an Open Access article distributed under the terms of the Creative Commons Attribution License (http://creativecommons.org/licenses/by/2.0), which permits unrestricted use, distribution, and reproduction in any medium, provided the original work is properly credited. assessment of gait and balance [3,4]. There are a number of performance orientated mobility assessment tools that assess aspects of balance and gait involved in normal daily activities. These tools serve to identify patients at risk of falling however, the sensitivity and specificity of existing tools is low [5]. One such example is the STRATIFY clinical prediction rule (St. Thomas Risk Assessment Tool in Falling elderly inpatients), which consists of five items that address risk factors for falling including past history of falling, patient agitation, visual impairment affecting everyday function, need for frequent toileting, and transfer ability and mobility. The STRATIFY rule yields a possible score between 0 and 5 (each item scoring 1 if present or 0 if absent). A recent systematic review examined the predictive value of the rule in elderly inpatients at risk of falls and found that at a score $\geq 2$ points, the STRATIFY rule had only limited predictive ability with moderate summary estimates of sensitivity $(0.67,95 \%$ CI $0.52-0.80)$ and specificity $(0.57,95 \%$ CI $0.45-0.69)$ [6]. 
The TUG test is another commonly used screening tool for falls risk in the inpatient and the community setting. The TUG (Timed Up and Go) test was developed in 1991 as a modified timed version of the Get up and Go test $[7,8]$. To perform the TUG test as described in the original derivation study, the patient is timed while they rise from an arm chair (approximate seat height $46 \mathrm{~cm}$ ), walk at a comfortable and safe pace to a line on the floor three metres away, turn and walk back to the chair and sit down again. The subject walks through the test once before being timed to become familiar with the test. The subject wears his regular footwear and uses his customary walking aid (cane or walker) if necessary [8]. A faster time indicates a better functional performance and a score of $\geq 13.5$ seconds is used as a cut-point to identify those at increased risk of falls in the community setting [9]. However, reported threshold values vary from 10 to 33 seconds in the literature [10,11].

The TUG is recommended as a routine screening test for falls in guidelines published by the American Geriatric Society and the British Geriatric Society [12]. The National Institute of Clinical Evidence (NICE) guidelines also advocate the use the TUG for assessment of gait and balance in the prevention of falls in older people [13]. To date three systematic reviews have examined the clinical utility of the TUG to discriminate between those at low and high risk of falling [14-16]. The most recent systematic review reported that the pooled mean difference in time taken to complete the TUG between fallers and non-fallers depended on the baseline functional status of the cohort of patients under investigation. In essence, there was a mean difference of 0.63 seconds (95\% CI 0.14-1.12 seconds) in the performance of the TUG for high-functioning versus a difference of 3.59 seconds (95\% CI $=2.18-4.99$ seconds) for those in institutional settings [16]. The aim of this systematic review with metaanalysis is to examine the predictive value of the test to identify individuals at risk in falling in the community using the frequently cited cut-off of $\geq 13.5$ seconds. A secondary aim of the study is to examine the summary estimates of sensitivity and specificity of alternative cut-off scores to optimally discriminate between fallers and non-fallers.

\section{Methods}

\section{Search strategy}

This systematic review and meta-analysis was performed according to the principles outlined by the Cochrane diagnostic test accuracy working group $[17,18]$. We aimed to identify all studies that validated the TUG test in community dwelling older adults. A systematic literature search was conducted in June 2012 (updated in March 2013) and included the following search engines: Pubmed, EMBASE, Cochrane Library, EBSCO, CINAHL and SCOPUS. A combination of the following keywords and $\mathrm{MeSH}$ terms were used: 'Timed Up and Go test', 'Get Up and Go test', 'TUG', 'GUG', 'TGUG', 'TGUGT', 'ETGUG', 'ETGUGT”,TUGT', 'modified TUG' and 'accidental falls', 'fall', 'falling', 'faller'. No language restrictions were applied to the search. The search was supplemented by hand searching reference lists of retrieved articles and searching Google scholar. The original version of the Get Up and Go test was created in 1986 [7], the timed version was later derived in 1991 [8], therefore only studies published from 1991-2013 were included in our literature review.

\section{Study selection and data extraction}

Studies were included if they met the following inclusion criteria: 1) Prospective or retrospective cohort studies or randomised control trials, 2) Studies that included community dwelling older adults as the population of interest, 3) Studies that validated the original version of the TUG test, 4) Studies that recorded a subsequent fall. Studies were excluded if their population of interest was limited to patients with specific neurological or orthopaedic condition e.g. Parkinson's disease, stroke, hip fracture or amputation of a lower limb. Studies were also excluded if they were limited to a population with a particular medical condition e.g. patients with chronic obstructive pulmonary disease. For the purposes of this review, we included studies where $\geq 80 \%$ of subjects were community dwelling and/ or were described as self caring or independent. Studies where $>20 \%$ of the subject population were described as institutionalised, living in nursing homes, residential care homes or geriatric inpatients were excluded. The definition of a subsequent fall was considered in the context of each individual study. We considered the following definition of a fall: 'an unexpected event in which the patient comes to rest on the ground, floor or lower level as the reference standard [1] and variations of this definition were recorded in Table 1 that contains details of the included studies.

Two reviewers (EB, RG) read the titles and/or abstracts of the identified references and eliminated irrelevant studies. Studies that were considered eligible for inclusion were read fully in duplicate and their suitability for inclusion was independently determined by both RG and EB. Disagreement was managed by consensus. Data were extracted on study type and setting, patient demographics (age, gender) and clinical characteristics including relevant inclusion and exclusion criteria, person who administered the TUG, person who recorded the subsequent fall, the definition of a fall used. For the purposes of this paper, the unit of analysis was the patient or "faller" rather than each "fall" to avoid duplication bias. Authors were contacted by email to provide further information on patient cohorts where there was insufficient data provided. Studies that included data on the same patient cohort for more than one publication were only included once in the meta-analysis. 


\section{Quality assessment}

The methodological quality of the selected studies was evaluated independently by two reviewers (EB and $\mathrm{FH}$ ) using the QUADAS-2 tool, a validated tool for the quality assessment of diagnostic accuracy studies $[17,18]$. This checklist consists of four key domains: patient selection, index test, reference standard and flow and timing. Within each study, the domains are assessed in terms of risk of bias and the first three of these domains are assessed in terms of concerns about applicability. Signalling questions as specified in the QUADAS-2 tool enable the reviewer to give each domain a rating of high, low or unclear. Disagreements were resolved by a third reviewer (RG).

\section{Statistical methods}

We used Stata version 12 (StataCorp College Station, Texas, USA), particularly the metandi command that fits the bivariate model, for all statistical analyses. We have applied this methodology in similar studies [19]. Significance was set at $\mathrm{p}<0.05$ for all analyses. A $2 \times 2$ table was constructed to extract the number of true positives, false positives, true negatives, false negatives, for the TUG test from each validation study using the pre-defined cut-point of $\geq 13.5$ seconds to identify those at increased risk of falling. We applied the bivariate random effects model to estimate summary estimates of sensitivity and specificity and their corresponding 95\% confidence intervals. This approach preserves the two-dimensional nature of the original data and takes into account both study size and the heterogeneity beyond chance between studies [20]. Sensitivity refers to the proportion of fallers correctly classified as high risk. Specificity is the proportion of non-fallers correctly classified as low risk.

The sensitivity and specificity for the TUG test was plotted in a hierarchical summary receiver operating characteristic (HSROC) graph, plotting sensitivity (true positive) on the y axis against 1-specificity (false negative) on the $\mathrm{x}$ axis. The $95 \%$ confidence region and the 95\% prediction region were plotted around pooled estimates to illustrate the precision with which the pooled values were estimated (confidence ellipse around the mean value) and to illustrate the amount of between study variation (prediction ellipse).

Heterogeneity was evaluated visually using the summary ROC plots and statistically by using the variance of logit transformed sensitivity and specificity, with smaller values indicating less heterogeneity between studies. Bayes' theorem was used to estimate the post test probability of a fall by multiplying the pre-test odds by the likelihood ratio; where pre-test odds are calculated by dividing the pre-test probability by (1+ pre-test probability) and the post -test probability equals post test odds divided by $(1+$ post-test odds). We completed sensitivity analyses to explore the effect of methodological features, as determined by the QUADAS-2 tool, on the predictive value of the TUG test.

The $c$ statistic, or area under the curve, with 95\% CI were also estimated to describe model discrimination. The $c$ statistic ranges from 0.5 (no discrimination) to a theoretical maximum of 1 , values between 0.7 and 0.9 represent moderate accuracy and greater than 0.9 represents high accuracy. A c statistic of 1 represents perfect discrimination, whereby scores for all cases (fallers) are higher than those for all the non-cases (non-fallers) with no overlap [21]. Finally, the association between the TUG score and falls was assessed using logistic regression and is presented as odds ratios with $95 \%$ confidence intervals.

\section{Results}

\section{Study identification}

A flow diagram of the search strategy is presented in Figure 1. Two researches (EB, RG) screened all potential papers. The search strategy yielded 1,134 articles and an additional 20 articles were found by hand searching resulting in 1,154 articles. Six hundred and fifty five articles remained after duplicates were removed. Five hundred and fifty were then excluded based on title or abstract. Of the remaining 105 articles, 80 were excluded after reading the full text leaving 25 articles. Within this group, there were four publications based on two unique cohorts of patients [22-25].

\section{Study characteristics}

The characteristics of the 25 prospective cohort studies are contained in Table 1. The descriptive characteristics were combined where two studies were based on the same population of patients [22-25]. In relation to 25 studies: seven studies were based in the USA [26-32], five in Japan [33-37], three in Israel [22,23,38], four in France $[24,25,39,40]$ and one in each of Taiwan [41], Australia [42], the UK (unpublished) [43], Brazil [44], Ireland [45] and Norway [46]. The size of the patient cohort in the included studies ranged from 13 [29] to 1618 patients [40]. In total 2,314 patients were included in the meta-analysis from 10 different datasets. The duration of follow up varied from six months $[27,28,31,32,34]$ to five years [33].

The application and the conditions of testing varied in many of the validation studies - variations included the instruction to walk as quickly as possible during the task $[26,46]$, the sole use or non-use of an assistive device [36,45] standing from an armless chair [27], seat height variations $40 \mathrm{~cm} \mathrm{[35]} \mathrm{to} 50 \mathrm{~cm}$ [41], walking with arms crossed [26]. Testing conditions also varied in that some studies to allow a practice attempt and/or record the average time of two or three attempts [28]. 
Table 1 Descriptive characteristics of the studies included in the review

\begin{tabular}{|c|c|c|c|c|c|c|c|}
\hline Study & $\begin{array}{l}\text { Participants }(n) \text { sex, } \\
\text { mean age }(+/- \text { SD) }\end{array}$ & $\begin{array}{l}\text { Time frame } \\
\text { of follow-up }\end{array}$ & $\begin{array}{l}\text { Person administering } \\
\text { test }\end{array}$ & Definition of falls & $\begin{array}{l}\text { Method of administering } \\
\text { the test }\end{array}$ & $\begin{array}{l}\text { Method of } \\
\text { recording falls }\end{array}$ & $\begin{array}{l}\text { Number of individuals } \\
\text { who fell }\end{array}$ \\
\hline \multirow{4}{*}{$\begin{array}{l}\text { Greene et al. } \\
2012^{*}\end{array}$} & $N=349$ & \multirow[t]{4}{*}{2 years } & \multirow[t]{4}{*}{ Not recorded } & \multirow[t]{4}{*}{ Tinetti 1997} & \multirow{4}{*}{$\begin{array}{l}\text { Test completed once. } \\
\text { Assistive device not } \\
\text { permitted. }\end{array}$} & \multirow{4}{*}{$\begin{array}{l}\text { Self report with collateral } \\
\text { information from relatives } \\
\text { and medical records }\end{array}$} & \multirow[t]{4}{*}{$N=83$} \\
\hline & $M=103$ & & & & & & \\
\hline & $F=246$ & & & & & & \\
\hline & $\begin{array}{l}\text { Mean age } \\
71.5+/-6.7\end{array}$ & & & & & & \\
\hline \multirow{4}{*}{$\begin{array}{l}\text { Herman et al. } \\
2011 \text { and } 2010\end{array}$} & $N=265$ & \multirow[t]{4}{*}{3 years } & \multirow[t]{4}{*}{ Physiotherapist } & \multirow{4}{*}{$\begin{array}{l}\text { Leveille } 2009 \text { and } \\
\text { Mackensie } 2006\end{array}$} & \multirow{4}{*}{$\begin{array}{l}\text { Test performed twice } \\
\text { (mean score used). } \\
\text { Assistive device } \\
\text { not permitted }\end{array}$} & \multirow{4}{*}{$\begin{array}{l}\text { Self report falls } \\
\text { diary monthly }\end{array}$} & \multirow[t]{2}{*}{$N=64$ (1 year follow up) } \\
\hline & $M=111$ & & & & & & \\
\hline & $F=154$ & & & & & & $N=131$ (2 year follow up) \\
\hline & $\begin{array}{l}\text { Mean age } \\
76.4+/-4.3\end{array}$ & & & & & & $N=73$ retrospective \\
\hline \multirow{4}{*}{$\begin{array}{l}\text { Russell et al. } \\
2008^{*}\end{array}$} & $N=344$ & \multirow[t]{4}{*}{1 year } & \multirow{4}{*}{$\begin{array}{l}\text { Physiotherapist, } \\
\text { occupational } \\
\text { therapist or } \\
\text { medical doctor }\end{array}$} & \multirow[t]{4}{*}{ Kellogg 1987} & \multirow[t]{4}{*}{ As per original TUG. } & \multirow{4}{*}{$\begin{array}{l}\text { Self report falls } \\
\text { diary bi-monthly }\end{array}$} & \multirow[t]{4}{*}{$N=164$} \\
\hline & $M=106$ & & & & & & \\
\hline & $F=238$ & & & & & & \\
\hline & $\begin{array}{l}\text { Mean age } \\
75.9+/-8.5\end{array}$ & & & & & & \\
\hline \multirow{3}{*}{$\begin{array}{l}\text { Viccaro et al. } \\
2011^{*}\end{array}$} & $N=457$ & \multirow[t]{3}{*}{1 year } & \multirow[t]{3}{*}{ Not recorded } & \multirow{3}{*}{$\begin{array}{l}\text { Defined as unintentionally } \\
\text { coming to rest on the } \\
\text { ground or other surface. }\end{array}$} & \multirow{3}{*}{$\begin{array}{l}\text { As per original TUG. } \\
\text { Average of } 2 \text { trials } \\
\text { reported. }\end{array}$} & \multirow[t]{3}{*}{ Interview at $3 / 12$ visit } & \multirow[t]{3}{*}{$N=174$} \\
\hline & $F=201 M=256$ & & & & & & \\
\hline & Mean age $=74$ & & & & & & \\
\hline \multirow{3}{*}{$\begin{array}{l}\text { Killough et al. } \\
2006^{*}\end{array}$} & $N=122$ & \multirow[t]{3}{*}{6 months } & \multirow[t]{3}{*}{ Unrecorded } & \multirow[t]{3}{*}{ Not defined } & Administered test only & Interviewed every & $N=$ not recorded \\
\hline & $M / F=$ unrecorded & & & & $\begin{array}{l}\text { once unless misunderstood } \\
\text { and a second trial was used. }\end{array}$ & 3 months by telephone & \\
\hline & $\begin{array}{l}\text { Mean age = } \\
\text { unrecorded }\end{array}$ & & & & & & \\
\hline Thomas & $N=31$ & 12 months & Physiotherapist & Kellogg 1987 & Shoe should have maximum & Self report by monthly & $\mathrm{N}=16$ had 2 or more falls, \\
\hline $\begin{array}{l}\text { unpublished } \\
\text { study* }\end{array}$ & $M=6$ & & & & $\begin{array}{l}\text { heel height of } 4 \mathrm{~cm} \text {. } 1 \text { UG not } \\
\text { described in any further detail. }\end{array}$ & $\begin{array}{l}\text { prepaid postcards and } \\
\text { follow up phone call }\end{array}$ & 15 had 1 \\
\hline & $F=25$ & & & & & at $3,6,9 / 12$ & \\
\hline & Mean age $=81.6$ & & & & & & \\
\hline Aoyama et al. & $N=58$ & 6 months & Physiotherapist & Tinetti 1988 & As per original TUG. Two test & Self report by falls diary, & $N=25$ \\
\hline & $F=58$ & & & & & & \\
\hline & $M=0$ & & & & & & \\
\hline & $\begin{array}{l}\text { Mean age } \\
80.5+/-5.7\end{array}$ & & & & & & \\
\hline
\end{tabular}


Table 1 Descriptive characteristics of the studies included in the review (Continued)

\begin{tabular}{|c|c|c|c|c|c|c|c|}
\hline \multirow{4}{*}{$\begin{array}{l}\text { Sai et al. } \\
2009^{*}\end{array}$} & $N=137$ & \multirow[t]{4}{*}{12 months } & \multirow[t]{4}{*}{ Trained clinical staff } & \multirow[t]{4}{*}{ Buchner 1993} & \multirow{4}{*}{$\begin{array}{l}\text { Time taken for subject to get } \\
\text { up from chair (with arms } \\
\text { crossed across chest) walk } 10 \mathrm{ft} \text {, } \\
\text { turn around and sit back down } \\
\text { as quickly as possible. }\end{array}$} & \multirow{4}{*}{$\begin{array}{l}\text { Self report by falls diary. } \\
\text { This was followed by } \\
\text { monthly phone-calls. }\end{array}$} & \multirow[t]{4}{*}{$N=70$} \\
\hline & $M=48$ & & & & & & \\
\hline & $F=89$ & & & & & & \\
\hline & $\begin{array}{l}\text { Mean age } \\
76.7+/-6.1\end{array}$ & & & & & & \\
\hline \multirow{4}{*}{$\begin{array}{l}\text { Alexandre et al. } \\
2012^{*}\end{array}$} & $N=63$ & \multirow[t]{4}{*}{12 months } & \multirow{4}{*}{$\begin{array}{l}\text { Trained Physical } \\
\text { therapists }\end{array}$} & \multirow{4}{*}{$\begin{array}{l}\text { Kellogg Working } \\
\text { Group } 1987\end{array}$} & \multirow{4}{*}{$\begin{array}{l}\text { As per original TUG seat } \\
\text { height } 42 \mathrm{~cm} \text {, back } 79 \mathrm{~cm} \text {, } \\
\text { arms } 60 \mathrm{~cm} \text { from ground. } \\
\text { Participants used own foot } \\
\text { wear and used assistive } \\
\text { device if needed. }\end{array}$} & \multirow{4}{*}{$\begin{array}{l}\text { Interviewed every } 3 / 12 \\
\text { by blinded evaluator and } \\
\text { self report via log book } \\
\text { collected every } 3 / 12 \text {. }\end{array}$} & \multirow[t]{4}{*}{$N=21$} \\
\hline & Male = 30 & & & & & & \\
\hline & Female $=33$ & & & & & & \\
\hline & $\begin{array}{l}\text { Mean age Fallers }= \\
66.68+/-5.57 . \text { Non } \\
\text { fallers }=66.36+/-4.60\end{array}$ & & & & & & \\
\hline \multirow{4}{*}{$\begin{array}{l}\text { Yamada et al. } \\
2010\end{array}$} & $N=171$ & \multirow[t]{4}{*}{1 year } & \multirow[t]{4}{*}{ Trained staff members } & \multirow[t]{4}{*}{ Koski 1996} & \multirow{4}{*}{$\begin{array}{l}\text { As per original TUG, height } \\
40 \mathrm{~cm}, 3 \mathrm{~m} \text { at normal pace } \\
\text { turn walk back to chair and } \\
\text { sit down. } 2 \text { trials average } \\
\text { time recorded }\end{array}$} & \multirow{4}{*}{$\begin{array}{l}\text { Monthly telephone } \\
\text { calls using structured } \\
\text { questionnaire. Self report } \\
\text { by mail every month. }\end{array}$} & \multirow[t]{4}{*}{$N=59$} \\
\hline & $F=134$ & & & & & & \\
\hline & $M=37$ & & & & & & \\
\hline & Age $80.5+/-5.6$ & & & & & & \\
\hline \multirow{8}{*}{$\begin{array}{l}\text { Yamada et al. } \\
2012\end{array}$} & $N=252$ & \multirow[t]{8}{*}{1 year } & \multirow[t]{8}{*}{ Trained researchers } & \multirow[t]{8}{*}{ Koski 1996} & \multirow{8}{*}{$\begin{array}{l}\text { Participants asked to stand up } \\
\text { from a standard chair seat } \\
\text { height } 40 \mathrm{~cm} \text {, walk a distance } \\
\text { of } 3 \mathrm{~m} \text { at a maximum pace, } \\
\text { turn walk back and sit down. } \\
\text { Better performance of two } \\
\text { attempts recorded. Walking } \\
\text { aid permitted }\end{array}$} & \multirow{8}{*}{$\begin{array}{l}\text { Interview at end } \\
\text { of follow up }\end{array}$} & \multirow[t]{8}{*}{$N=71$} \\
\hline & $\begin{array}{l}231 \text { who completed } \\
\text { study: }\end{array}$ & & & & & & \\
\hline & Male = 54 & & & & & & \\
\hline & Female = 177 & & & & & & \\
\hline & Mean age $=$ & & & & & & \\
\hline & $\mathrm{T} 1=73.9+/-6.6$ & & & & & & \\
\hline & $\mathrm{T} 2=79.1+/-7.0$ & & & & & & \\
\hline & $\mathrm{T} 3=82.0+/-6.9$ & & & & & & \\
\hline \multirow{4}{*}{$\begin{array}{l}\text { Wrisley et al. } \\
2010\end{array}$} & $N=35$ & \multirow[t]{4}{*}{6 months } & \multirow[t]{4}{*}{ Physical therapist } & \multirow{4}{*}{$\begin{array}{l}\text { Defined as unintentional } \\
\text { contact below pt's height } \\
\text { and classified as unexplained } \\
\text { or unexplained. A fall } \\
\text { was considered explained } \\
\text { if there was medical } \\
\text { environmental or task-related } \\
\text { explanation for the fall } \\
\text { that was unavoidable. } \\
\text { An unexplainable fall } \\
\text { was all other falls. }\end{array}$} & \multirow{4}{*}{$\begin{array}{l}\text { As per original TUG. } \\
\text { Participants were } \\
\text { allowed } 1 \text { practice } \\
\text { trial and then preformed } \\
3 \text { timed trials. The average } \\
\text { of } 3 \text { trials reported }\end{array}$} & Self report by daily & $\mathrm{N}=17$ (6 participants \\
\hline & $\begin{array}{l}\text { Mean age = } \\
72.9+/-7.8\end{array}$ & & & & & $\begin{array}{l}\text { a separate postcard } \\
\text { providing details of }\end{array}$ & \\
\hline & $M=17$ & & & & & $\begin{array}{l}\text { any falls with follow } \\
\text { up phone call }\end{array}$ & \\
\hline & $\mathrm{F}=18$ & & & & & & \\
\hline
\end{tabular}


Table 1 Descriptive characteristics of the studies included in the review (Continued)

\begin{tabular}{|c|c|c|c|c|c|c|c|}
\hline \multirow{4}{*}{$\begin{array}{l}\text { Pai et al. } \\
2010\end{array}$} & $N=13$ & \multirow[t]{4}{*}{1 year } & \multirow[t]{4}{*}{ Not recorded } & \multirow{4}{*}{$\begin{array}{l}\text { Defined as any event } \\
\text { in which they landed } \\
\text { unintentionally on a } \\
\text { lower surface such as a } \\
\text { chair, the floor or ground }\end{array}$} & \multirow{4}{*}{$\begin{array}{l}\text { As per original TUG, } \\
\text { one practice trial given. }\end{array}$} & \multirow{4}{*}{$\begin{array}{l}\text { Contacted by telephone } \\
\text { between } 29-32 \text { months } \\
\text { into study }\end{array}$} & \multirow[t]{4}{*}{$N=4$} \\
\hline & $M=9$ & & & & & & \\
\hline & $\mathrm{F}=4$ & & & & & & \\
\hline & Mean age $=72+/-5$ & & & & & & \\
\hline \multirow{4}{*}{$\begin{array}{l}\text { Okumiya et al. } \\
1998\end{array}$} & $N=328$ & \multirow{4}{*}{$\begin{array}{l}5 \text { years } \\
\text { prospective }\end{array}$} & \multirow[t]{4}{*}{ Not recorded } & \multirow[t]{4}{*}{ Not defined } & \multirow[t]{4}{*}{ As per original TUG } & \multirow{4}{*}{$\begin{array}{l}\text { Self-administered } \\
\text { questionnaire }\end{array}$} & \multirow[t]{4}{*}{68} \\
\hline & $M=151$ & & & & & & \\
\hline & $F=177$ & & & & & & \\
\hline & Mean age $=80.3$ & & & & & & \\
\hline \multirow{4}{*}{$\begin{array}{l}\text { Lin et al. } \\
2004\end{array}$} & $N=1200$ & \multirow[t]{4}{*}{1 year } & \multirow[t]{4}{*}{ Trained interviewer } & \multirow[t]{4}{*}{ Not defined } & \multirow{4}{*}{$\begin{array}{l}\text { As per original TUG } \\
\text { standard chair with } \\
\text { seat height of } \\
40-50 \mathrm{~cm} \text { height }\end{array}$} & \multirow{4}{*}{$\begin{array}{l}\text { Self report by postcard } \\
\text { when a fall occurred and } \\
\text { telephoned every } 3 / 12 \text {. }\end{array}$} & \multirow[t]{4}{*}{ Not recorded } \\
\hline & $M=709$ & & & & & & \\
\hline & $F=491$ & & & & & & \\
\hline & Mean age $=73.4$ & & & & & & \\
\hline \multirow{4}{*}{$\begin{array}{l}\text { Buatois et al. } \\
2006\end{array}$} & $N=206$ & \multirow[t]{4}{*}{16 months } & \multirow[t]{4}{*}{ Not recorded } & \multirow[t]{4}{*}{ Tinetti 1988} & \multirow[t]{4}{*}{ As per original TUG } & \multirow{4}{*}{$\begin{array}{l}\text { Self report by } \\
\text { falls calendar } \\
\text { and questionnaire }\end{array}$} & \multirow[t]{4}{*}{$N=57$} \\
\hline & $M=116$ & & & & & & \\
\hline & $F=90$ & & & & & & \\
\hline & Mean age $=70+/-4$ & & & & & & \\
\hline \multirow{4}{*}{$\begin{array}{l}\text { Buatois et al. } \\
2010\end{array}$} & $N=1618$ & \multirow{4}{*}{$\begin{array}{l}\text { Mean time } \\
25+/-5 \text { months } \\
\text { (18-36 months) }\end{array}$} & \multirow[t]{4}{*}{ Not recorded } & Tinetti 1999 & As per original TUG & Self report by & $N=333$ \\
\hline & $M=821$ & & & & & $\begin{array}{l}\text { questionnaire at end } \\
\text { of study. Mean follow up }\end{array}$ & \\
\hline & $F=797$ & & & & & period $25+/-5$ months & \\
\hline & Mean age 70 & & & & & & \\
\hline $\begin{array}{l}\text { Bergland et al. } \\
2003^{*}\end{array}$ & $N=307$ & 1 year & Not recorded & $\begin{array}{l}\text { Falls defined as an } \\
\text { unintentional change } \\
\text { in position resulting in } \\
\text { the victim lying on the } \\
\text { floor or on the ground. }\end{array}$ & $\begin{array}{l}\text { The subject was instructed } \\
\text { to rise from a chair, walk } \\
3 \mathrm{~m} \text { as quickly as possible, } \\
\text { cross a line, turn, walk back, } \\
\text { and sit down again. }\end{array}$ & $\begin{array}{l}\text { Self report by daily } \\
\text { calendar, to return } \\
\text { calendar every } 3 / 12 \text { with } \\
\text { follow up phone call if a } \\
\text { fall occurred. Subjects }\end{array}$ & 155 \\
\hline & $M=0$ & & & & Wearing ordinary shoes & calendar were contacted & \\
\hline & $F=307$ & & & & walking aids if needed. & & \\
\hline & Mean age 80.8 & & & & & & \\
\hline Trueblood et al. & $N=198$ & 6 months & Researcher & Anacker 1992 & As per TUG, armless chair, & Telephone survey 4 & $N=30$ \\
\hline & $M=38$ & & & & $\begin{array}{l}3 \text { timed trials average } \\
\text { time recorded }\end{array}$ & $\begin{array}{l}\text { and } 6 \text { months using } \\
\text { a formalised script. }\end{array}$ & \\
\hline & $F=160$ & & & & & & \\
\hline & $\begin{array}{l}\text { Mean age } \\
78.1+/-8.2\end{array}$ & & & & & & \\
\hline
\end{tabular}


Table 1 Descriptive characteristics of the studies included in the review (Continued)

\begin{tabular}{|c|c|c|c|c|c|c|c|}
\hline \multirow{4}{*}{$\begin{array}{l}\text { Shimada et al. } \\
2009\end{array}$} & $N=445$ & \multirow[t]{4}{*}{1 year } & \multirow{4}{*}{$\begin{array}{l}\text { Day centre staff } \\
\text { nursing allied health } \\
\text { or similar qualifications }\end{array}$} & \multirow{4}{*}{$\begin{array}{l}\text { Nevitt } 1989 \text { Cumming } \\
2008\end{array}$} & \multirow{4}{*}{$\begin{array}{l}\text { As per TUG, measured } \\
\text { once at usual pace } \\
\text { assistive device allowed }\end{array}$} & \multirow{4}{*}{$\begin{array}{l}\text { Self report questionnaire, } \\
\text { with collateral if difficulty } \\
\text { in recall }\end{array}$} & \multirow[t]{4}{*}{$N=99$} \\
\hline & $F=310$ & & & & & & \\
\hline & $M=135$ & & & & & & \\
\hline & $\begin{array}{l}\text { Mean age } \\
80.5+/-7.2\end{array}$ & & & & & & \\
\hline \multirow{4}{*}{$\begin{array}{l}\text { Melzer et al. } \\
2009^{*}\end{array}$} & $N=100$ & \multirow{4}{*}{$\begin{array}{l}1 \text { year } \\
\text { prospective }\end{array}$} & \multirow[t]{4}{*}{ Research assistant } & \multirow[t]{4}{*}{ Tinetti 1988} & \multirow{4}{*}{$\begin{array}{l}\text { Not described in detail } \\
\text { but referenced as per } \\
\text { AGS/BGS which in turn } \\
\text { references Podsiadlo }\end{array}$} & \multirow{4}{*}{$\begin{array}{l}\text { Self report by daily } \\
\text { calendar. Contacted by } \\
\text { research assistant at } \\
\text { one month intervals } \\
\text { to monitor falls. }\end{array}$} & \multirow[t]{4}{*}{$N=49$} \\
\hline & Male $=26$ & & & & & & \\
\hline & Female $=72$ & & & & & & \\
\hline & $\begin{array}{l}\text { Mean age } \\
78.4+/-5.7\end{array}$ & & & & & & \\
\hline \multirow{4}{*}{$\begin{array}{l}\text { Beauchet et al. } \\
2007 \text { and } 2008\end{array}$} & $N=187$ & \multirow[t]{4}{*}{1 year } & \multirow[t]{4}{*}{ Trained evaluator } & \multirow{4}{*}{$\begin{array}{l}\text { Defined as } \\
\text { unintentionally } \\
\text { coming to rest } \\
\text { on the ground, } \\
\text { floor or lower } \\
\text { level }\end{array}$} & \multirow{4}{*}{$\begin{array}{l}\text { Not described } \\
\text { reference to } \\
\text { Podsiadlo }\end{array}$} & \multirow{4}{*}{$\begin{array}{l}\text { Monthly phone call } \\
\text { using a standardised } \\
\text { questionnaire. Collateral } \\
\text { obtained if cognitive } \\
\text { impairment }\end{array}$} & \multirow[t]{4}{*}{$N=54$} \\
\hline & $M=29$ & & & & & & \\
\hline & $F=158$ & & & & & & \\
\hline & $\begin{array}{l}\text { Mean Age }= \\
84.8+/-5.2\end{array}$ & & & & & & \\
\hline \multirow{4}{*}{$\begin{array}{l}\text { Garber et al. } \\
2010\end{array}$} & $N=904$ & \multirow[t]{4}{*}{6 months } & \multirow{4}{*}{$\begin{array}{l}\text { Trained bilingual } \\
\text { field interviewers }\end{array}$} & \multirow[t]{4}{*}{ Not defined } & \multirow{4}{*}{$\begin{array}{l}\text { Armless chair } 3 \mathrm{~m} \text { as } \\
\text { quickly } 2 \text { trials -first } \\
\text { practice second } \\
\text { recorded time }\end{array}$} & \multirow[t]{4}{*}{ Not recorded } & \multirow[t]{4}{*}{ Not recorded } \\
\hline & Male $=263$ & & & & & & \\
\hline & Female $=641$ & & & & & & \\
\hline & $\begin{array}{l}\text { Mean age } \\
76.6+/-0.5\end{array}$ & & & & & & \\
\hline \multicolumn{8}{|c|}{ 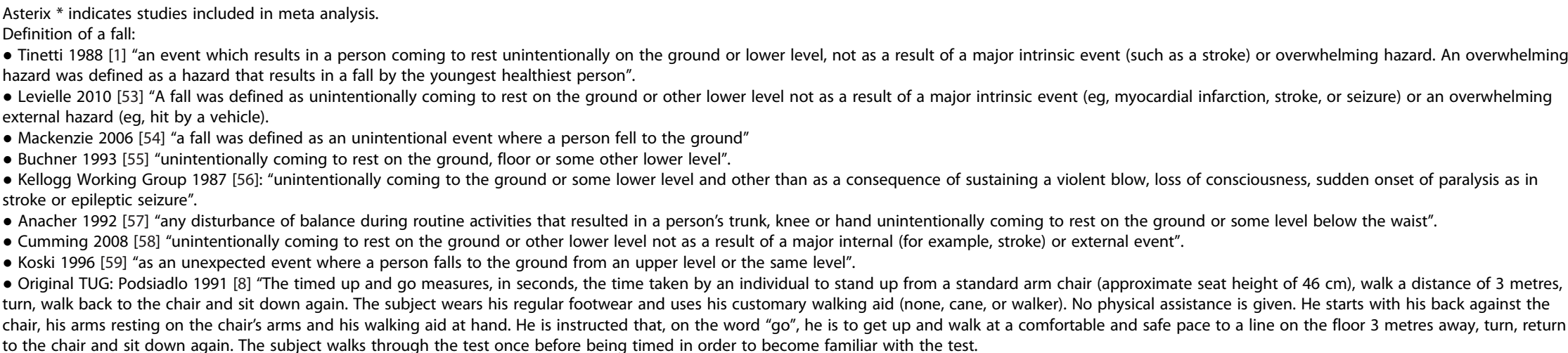 } \\
\hline
\end{tabular}




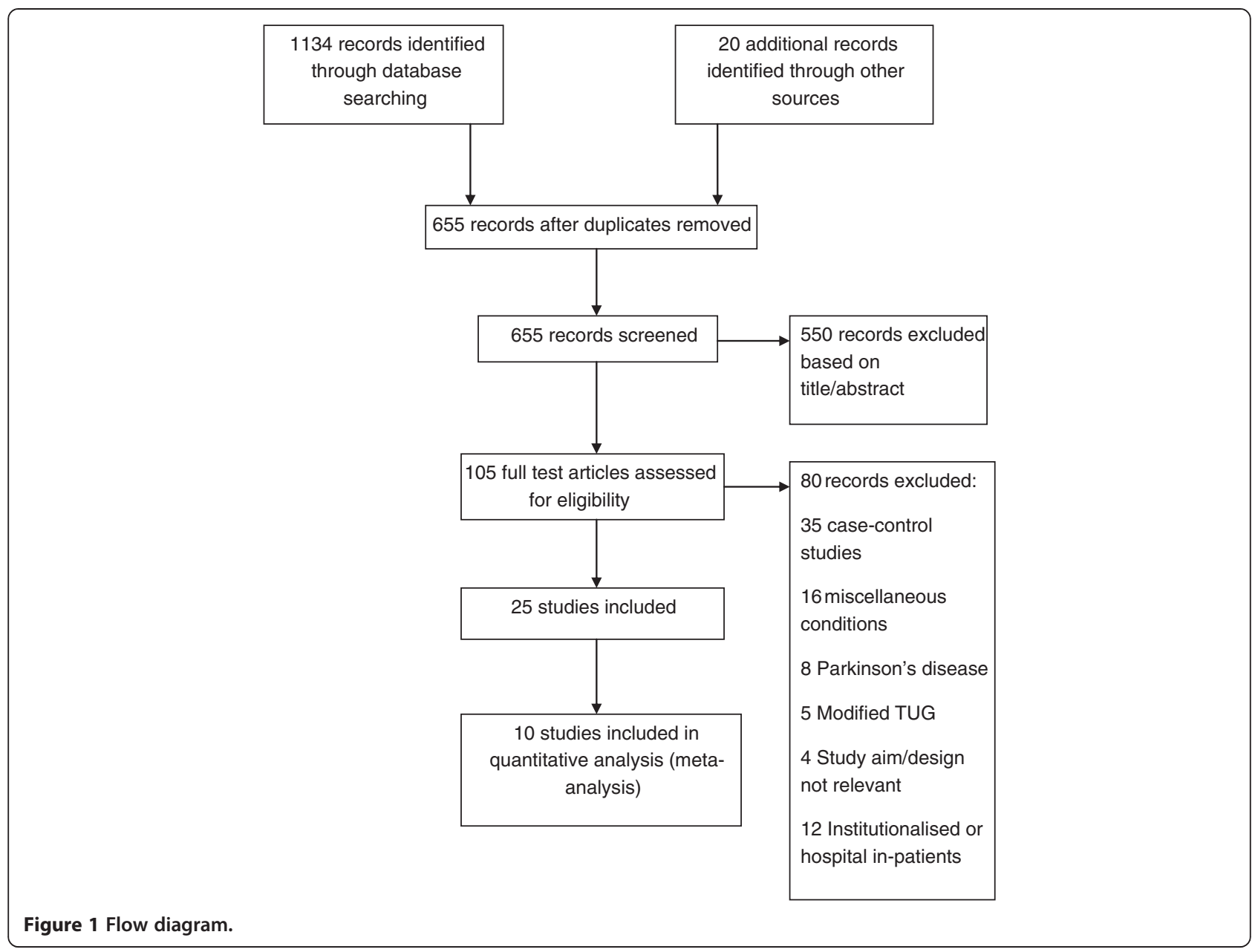

\section{Study quality}

The summary diagram of the quality assessment is shown in Figure 2. All twenty five articles were quality assessed. The overall quality of the studies included was moderate with six studies [23,27,30,35,36,39] rated as low in all domains in both risk of bias and concerns about applicability. Ten studies [22,24-26,31,33,37,38,41,43] rated as having an unclear risk of bias and nine studies $[28,29,32,34,40,42,44-46]$ were rated as having a high risk of bias. This was primarily attributed to a lack of information provided with respect to methods of patient recruitment (selection bias) and criteria used to ascertain of a subsequent fall (reference standard).

In relation to concerns about applicability of each individual study to the proposed research question, ten studies [23,26,27,30,35,36,39,40,42,45] were rated as low, ten studies $[22,24,25,29,33,37,38,41,43,44]$ were rated as unclear and five studies $[28,31,32,34,46]$ were considered as having high level of concern. A high or unclear risk of bias was noted in studies that inadequately described loss to follow-up in the cohort or the methods used record the incidence of a fall over the period of study.
The index test was adequately described in the majority of studies but the many studies failed to record whether the index test (TUG score) was interpreted with or without knowledge of the reference standard (subsequent fall).

\section{Predictive accuracy of all included studies}

All authors were contacted to request primary data and ten authors responded with the relevant data $[26,30,31,34,38,42-46]$. In two of the ten studies where data was provided $[26,46]$, the TUG was administered as quickly as possible and in the remaining eight studies it was administered at a comfortable pace. The duration of follow-up in these studies varied from six months $[31,34]$ to two years [45]. The remaining seven studies followed patients for one year after administration of TUG $[26,30,38,42-44,46]$. The pooled sensitivity, specificity and the respective variance of the logit transformed sensitivity and specificity for the ten studies included in the meta-analysis are displayed in Table 2 . These findings indicate that the TUG test is more useful at ruling in rather than ruling out falls in individuals classified as high risk ( $\geq 13.5$ seconds), with a higher pooled specificity 


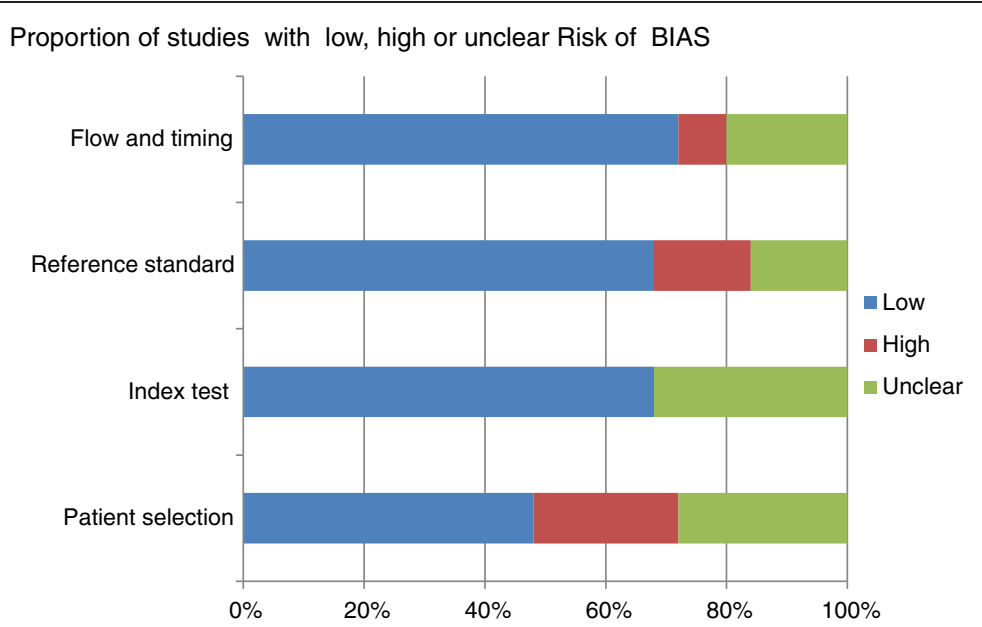

Proportion of studies with low, high, or unclear concerns regarding APPLICABILITY

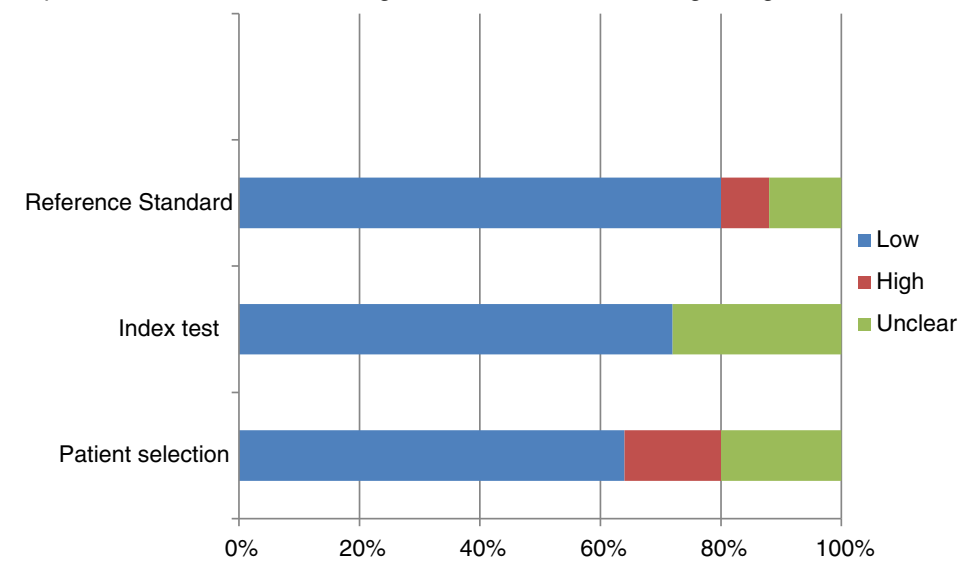

Figure 2 Methodological quality of the studies included in the review.

(0.73, 95\% CI $0.51-0.88)$ than sensitivity $(0.32,95 \%$ CI 0.14-0.57). Individual and summary estimates of sensitivity and specificity for all studies, the 95\% confidence region and $95 \%$ prediction region are presented in the summary ROC graph (Figure 3). The 95\% confidence region is broad, reducing the precision of studies in the pooled estimate. The 95\% prediction region (amount of variation between studies) is also wide suggesting heterogeneity between studies.

The logistic regression analysis also indicates that the TUG score is not a significant predictor of falls $(\mathrm{OR}=1.01,95 \% \mathrm{CI} 1.00-1.02, \mathrm{p}=0.04)$. The limited

Table 2 Summary estimates of sensitivity, specificity, and positive and negative likelihood ratios for all included studies and for sensitivity analyses at a cut point of $\geq 13.5$ seconds

\begin{tabular}{|c|c|c|c|c|c|}
\hline Application of TUG test & $\begin{array}{l}\text { No. of studies } \\
\text { (patients) }\end{array}$ & $\begin{array}{l}\text { Sensitivity } \\
(95 \% \mathrm{Cl})\end{array}$ & $\begin{array}{c}\text { Variance logit } \\
\text { sensitivity }(95 \% \mathrm{CI})\end{array}$ & $\begin{array}{l}\text { Specificity } \\
(95 \% \mathrm{Cl})\end{array}$ & $\begin{array}{c}\text { Variance logit } \\
\text { specificity }(95 \% \mathrm{Cl})\end{array}$ \\
\hline All studies & $10(n=2,314)$ & $0.32(0.14-0.57)$ & $2.62(0.94-7.29)$ & $0.73(0.51-0.88)$ & $2.24(0.76-6.63)$ \\
\hline $\begin{array}{l}\text { Studies where TUG was administered as } \\
\text { fast as possible excluded }\end{array}$ & $8(n=1,872)$ & $0.44(0.20-0.71)$ & $2.52(0.78-8.1)$ & $0.71(0.49-0.86)$ & $1.7(0.52-5.56)$ \\
\hline $\begin{array}{l}\text { Studies with duration of follow up }> \\
\text { or < one year excluded }\end{array}$ & $7(n=1,858)$ & $0.33(0.11-0.68)$ & $3.58(1.07-12.0)$ & $0.70(0.37-0.90)$ & $3.33(0.89-12.49)$ \\
\hline Studies with selection bias excluded & $6(n=1,253)$ & $0.29(0.10-0.60)$ & $2.31(0.56-9.56)$ & $0.64(0.20-0.93)$ & $5.11(0.86-30.47)$ \\
\hline $\begin{array}{l}\text { Studies with unclear/no details on index } \\
\text { test excluded }\end{array}$ & $6(n=1,636)$ & $0.33(0.17-0.54)$ & $1.05(0.30-3.63)$ & $0.71(0.58-0.81)$ & $0.46(0.12-1.65)$ \\
\hline Studies with unclear/no definition 'fall' excluded & $6(n=1,750)$ & $0.28(0.11-0.54)$ & $1.82(0.55-6.05)$ & $0.81(0.64-0.91)$ & $1.10(0.32-3.73)$ \\
\hline
\end{tabular}




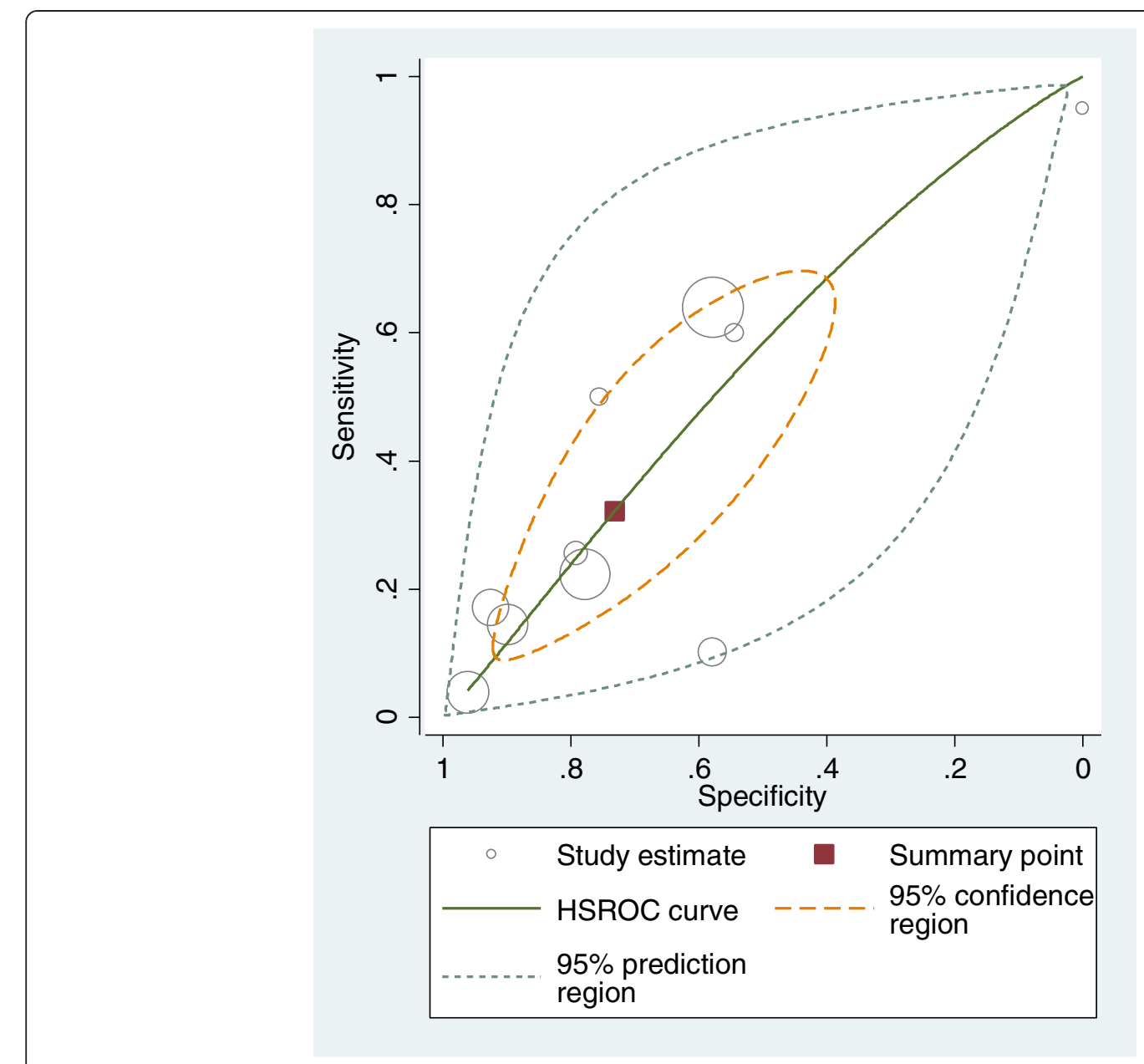

Figure 3 Hierarchical summary receiver operating characteristic plot of sensitivity and specificity for the TUG predicting falls at a cut point $\geq 13.5$ seconds.

discriminative performance of the TUG is confirmed by the ROC curve analysis (Figure 4) indicating about $57 \%$ overall accuracy by a significant area under the curve $\left(\mathrm{AUC}_{\mathrm{ROC}}=0.57,95 \%\right.$ CI 0.54-0.59).

\section{Sensitivity analysis}

A sensitivity analysis was completed excluding the two studies where the TUG was administered as fast as possible. $[26,46]$ The summary estimates of sensitivity $(0.44,95 \%$ CI $0.20-0.71)$ and specificity $(0.71,95 \%$ CI $0.49-0.86)$ were broadly unchanged. The three studies where the duration of follow up was less than or greater than one year were removed $[31,34,45]$. Similarly, the summary estimates of sensitivity $(0.33,95 \%$ CI $0.11-0.68)$ and specificity (0.70, 95\% CI 0.37-0.90) were unchanged. We also excluded four studies where there was evidence of selection bias $[34,42,44,46]$. Removal of these studies from the meta-analysis reduced the precision of the estimates of sensitivity $(0.29,95 \%$ CI $0.10-0.60)$ and specificity (0.64, 95\% CI $0.20-0.93)$. Four studies $[31,38,43,46]$ that did not adequately describe the method of administration of the TUG test were removed from the sensitivity analysis and the summary estimates of sensitivity $(0.33,95 \%$ CI $0.17-0.54)$ and specificity $(0.71,95 \%$ CI $0.58-0.81)$ of the TUG was broadly similar to the overall analysis. Finally we excluded four studies $[26,31,43,45]$ where no clear definition of a fall was reported. While the sensitivity remained stable $(0.28,95 \%$ CI $0.11-0.54)$, the predictive ability of the TUG to rule in individuals at high risk of falling increased to $81 \%$ (95\% CI 0.64-0.91). The pooled sensitivity, specificity and the respective variance of the logit transformed sensitivity and specificity for the studies included in the sensitivity analysis are displayed in Table 2.

\section{Bayesian analysis}

Using Bayes' theorem, the post-test probability of a fall across the different subgroups is presented in Table 3 . The pre-test probability (prevalence) was calculated as $51 \%$ across all studies. The cut-point of $\geq 13.5$ seconds has little impact on identifying those at high risk of falls 


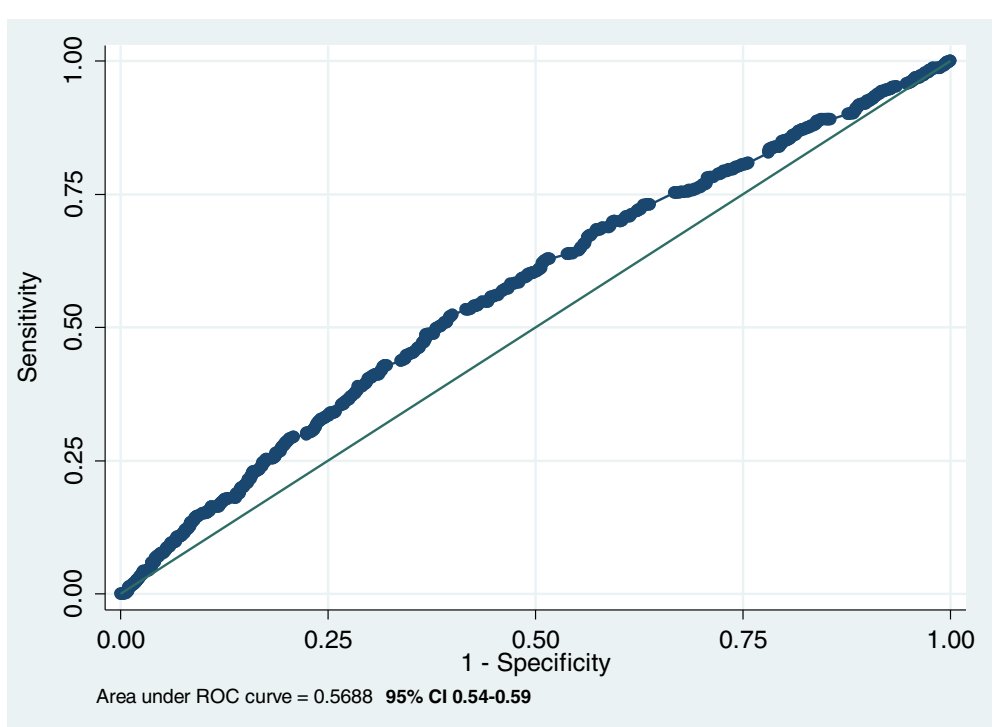

Figure 4 Performance of TUG to distinguish fallers from non-fallers.

when all studies are combined and across all of the different subgroups. Of note, when studies that provided no/unclear definitions of falls were excluded, the positive likelihood ratio increased to 1.50 (95\% CI 1.15-1.94) and the post-test probability of a fall in patients classified as high risk increased from $54 \%$ to $64 \%$.

\section{Discussion}

\section{Statement of principal findings}

This systematic review demonstrates that the diagnostic accuracy of the Timed Up and Go test is limited at the widely used cut point of $\geq 13.5$ seconds and should not be used for identifying community dwelling adults at high risk of falls in clinical practice. The sensitivity analysis which examined the performance of the rule in different subgroups also showed broadly comparable results, indicating that the TUG performed in a similar manner regardless of the methodological quality of the studies.
Results in the context of the current literature

The TUG is commonly used in the research and clinical setting to screen individuals at increased risk of falling. The commonly cited cut-off score of $\geq 13.5$ seconds used to identify individuals at high risk of falls was first described by Shumway-Cook and colleagues in 2000 [47]. However, the nature of the study design (case-control) used to derive the TUG was not optimal and subject to bias in terms of choosing appropriate controls and determining exposure. In addition, the study comprised of small numbers of patients with 15 fallers and 15 non-fallers included in the analysis. The definition of a fall was broad "any unplanned unexpected contact with a supporting surface, excluding unavoidable environmental hazards" and the study excluded those who had had one or fewer falls in the previous six months. The authors reported a sensitivity of $80 \%$ and specificity of $100 \%$, suggesting that the TUG is more useful at 'ruling-in' falls in those classified

Table 3 Post-test probability of a fall in patients classified as high risk ( $\geq 13.5$ seconds) and low risk ( $<13.5$ seconds) using the TUG score

\begin{tabular}{|c|c|c|c|c|c|}
\hline Application of TUG test & $\begin{array}{c}\text { Pre test } \\
\text { probability (\%) }\end{array}$ & + LR $(95 \% \mathrm{Cl})$ & $\begin{array}{l}\text { Post test probability } \\
(\%)+\text { LR }\end{array}$ & -LR (95\% Cl) & $\begin{array}{l}\text { Post test probability } \\
(\%)-L R\end{array}$ \\
\hline All studies & $51 \%(49 \%-53 \%)$ & $1.20(0.82-1.75)$ & $56 \%(54 \%-58 \%)$ & $0.93(0.78-1.10)$ & $49 \%(47 \%-51 \%)$ \\
\hline $\begin{array}{l}\text { Studies where TUG was administered } \\
\text { as fast as possible excluded }\end{array}$ & $52 \%(49 \%-54 \%)$ & $1.53(1.31-1.79)$ & $62 \%(59 \%-64 \%)$ & $0.79(0.62-1.01)$ & $46 \%(44 \%-48 \%)$ \\
\hline $\begin{array}{l}\text { Studies with duration of follow up }> \\
\text { or }<\text { one year excluded }\end{array}$ & $53 \%(51 \%-56 \%)$ & $1.11(0.64-1.92)$ & $56 \%(54 \%-59 \%)$ & $0.95(0.73-1.24)$ & $52 \%(50 \%-55 \%)$ \\
\hline Studies with selection bias excluded & $52 \%(50 \%-55 \%)$ & $0.81(0.40-1.16)$ & $47 \%(45 \%-50 \%)$ & $1.10(0.72-1.71)$ & $54 \%(52 \%-57 \%)$ \\
\hline $\begin{array}{l}\text { Studies with unclear/no details on index } \\
\text { test excluded }\end{array}$ & $56 \%(54 \%-58 \%)$ & $1.14(0.67-1.91)$ & $59 \%(57 \%-61 \%)$ & $0.95(0.74-1.21)$ & $55 \%(53 \%-57 \%)$ \\
\hline $\begin{array}{l}\text { Studies with unclear/no definition } \\
\text { 'fall' excluded }\end{array}$ & $54 \%(51 \%-56 \%)$ & $1.50(1.15-1.94)$ & $64 \%(61 \%-66 \%)$ & $0.89(0.75-1.04)$ & $51 \%(48 \%-53 \%)$ \\
\hline
\end{tabular}


as high risk. However, these findings need to be interpreted in the context of the methodological limitations of the study.

This systematic review only included cohort studies and randomised controlled trials where the index test (TUG) preceded the outcome of interest (fall) and the findings are in keeping with those reported in a previous systematic review that included both case-control and cohort studies [16]. The authors reported that the predictive accuracy of the TUG in identifying fallers across the included studies was poor to moderate and sensitivity and specificity were often close to chance [16]. Furthermore, cut-off points for identifying patients at increased risk of falls in independent-living patients varied between 8.1-16 seconds for performing TUG at a comfortable speed and between 11-13.5 seconds at a fast walking speed.

The limited predictive value of the TUG may be explained by the fact that the TUG is a single test which reflects strength balance and mobility nonetheless, the risk of falling has been shown to depend on multiple intrinsic and extrinsic factors $[48,49]$. The TUG does not appear to adequately encompass these risk factors. Recent literature has focused on the addition of a second manual [50] or cognitive task [51]. Nevertheless, the predictive ability of the tool remains limited with the inclusion of these tasks. Further study of the constituent parts of the TUG by quantifying body movements through the use of body worn sensors have increased the predictive accuracy of the TUG to almost $80 \%$ in one study [45].

\section{Strengths and weaknesses of the study}

This study pooled data from a broad range of studies enhancing the generalisability of its findings. We examined the methodological quality of the studies using the validated QUADAS-2 tool for assessing the quality of such studies. In addition, sensitivity analyses examined the effect of important methodological variables including studies with selection bias, index test bias and reference standard bias. We also used individual patient data rather than aggregate data to calculate summary estimates of sensitivity and specificity and their corresponding 95\% confidence intervals. This allowed more accurate data analysis by accounting for heterogeneity between studies and influences of sample size. However, the findings from the systematic review need to be interpreted in the context of the study limitations. Significant heterogeneity exists between the validation studies with respect to variation in the application of the TUG and a lack of information relating to the conditions of performing the TUG e.g. shoes worn, floor surface, chair seat and arm height, walk to a line or an X on floor. Studies have shown that these factors can affect TUG performance, time to complete the test was found to be significantly longer when a chair without armrests was used [52]. Studies varied in the number of practice trials given and an average result recorded. In other studies up to three attempts were given before a timed trial was done. Furthermore, some studies did not allow the use of assistive walking device and others specified the flooring type which has also been shown to affect TUG score.

\section{Clinical implications}

Falls risk screening tools are an important element of falls prevention in the community. It is necessary to identify patients at high risk for falls and to facilitate the effective delivery of appropriate interventions to such patients. Inaccuracy of falls screening tools leads to inappropriate distribution of resources, contributing to varying degrees of success and failure of falls prevention strategies. It is essential to establish the accuracy of such tools and identify alternative tools that may be able to identify patients at risk of falling more accurately. Despite a growing body of evidence indicating its limited ability to predict falls, the TUG continues to be mentioned in clinical guidelines as a potential tool to identify fallers [12,13]. This is most likely because it is easy and quick to perform and does not require specialist equipment. Nonetheless, the totality of evidence to date is that it has limited predictive ability and should not be used in isolation to identify community-dwelling older people at increased risk of falls. Clinicians who assess the elderly for risk of falling should ideally do so in a comprehensive manner, taking into account the multi-factorial nature of falls rather than relying on a single test of mobility.

\section{Areas of future research}

This study demonstrates that the TUG should no longer be used as a falls risk assessment in community dwelling elderly people. Gait, balance and to a lesser degree vision and cognition are inherently assessed in the TUG however, it does not include other accepted risk factors for falls e.g. medication use and morbidity. Further research is needed to determine its usefulness in lower functioning groups and those who have specific deficits in the areas of balance and mobility.

Advancing age is a primary risk factor for falling and recent studies have shown that the rate of falling remains at approx 30\% in older people since 1988 [2]. The reasons why the elderly fall continues to be explored in the literature, and further research is required to develop a comprehensive falls risk tool that can accurately identify the common risk factors that predict falls. In order to prevent falls and reduce the overall rate of falling in the elderly, falls prevention programmes should then be tailored to the individual needs of the patient. 


\section{Conclusions}

It is well recognised that falls assessment and prevention programmes are multi-factorial. Evidence from this systematic review of diagnostic accuracy suggests that a single assessment tool like the TUG should not be used to identify community dwelling older adults at increased risk of falls.

\section{Competing interests}

The authors declare that they have no competing interests.

\section{Authors' contributions}

All authors were involved in the study conception and design. EB performed a systematic search of the literature. Both EB and RG screened potential articles, EB and FH evaluated the methodological quality of studies, RG, EB and CK acquired data for analysis, performed statistical analysis and interpretation of data and drafted the paper. TF critically revised the draft manuscript. All authors read and approved the final manuscript.

\section{Acknowledgements}

We would like to gratefully acknowledge the data contributed by the following authors: T Alexandre, B Greene, T Herman, M Aoyama, A Bergland, M Russell, L Viccaro, J Killough, J Thomas, AJ Sai.

\section{Funding sources}

This work was supported by the Health Research Board (HRB) of Ireland through the HRB Centre for Primary Care Research under Grant HRC/2007/1.

Received: 27 August 2013 Accepted: 27 January 2014

Published: 1 February 2014

\section{References}

1. Tinetti ME, Speechley M, Ginter SF: Risk factors for falls among elderly persons living in the community. N Eng J Med 1988, 319(26):1701-1707.

2. Rubenstein LZ: Falls in older people: epidemiology, risk factors and strategies for prevention. Age Ageing 2006, 35(2):ii37-ii41.

3. Bloem BR, Steijns JA, Smits-Engelsman BC: An update on falls. Curr Opin Neurol 2003, 16(1):15-26.

4. Ganz DA, Bao Y, Shekelle PG, Rubenstein LZ: Will my patient fall? JAMA 2007, 297(1):77-86,

5. Oliver D, Daly F, Martin FC, MCMurdo ME: Risk factors and risk assessment tools for falls in hospital in-patients: a systematic review. Age Ageing 2004, 33(2):122-130.

6. Billington J, Fahey T, Galvin R: Diagnostic accuracy of the STRATIFY clinical prediction rule for falls: a systematic review and meta-analysis. BMC Fam Pract 2012, 13:76.

7. Mathias S, Nayak US, Isaacs B: Balance in elderly patients: the "Get-up and Go" test. Arch Phys Med Rehabil 1986, 67(6):387-389.

8. Podsiadlo D, Richardson S: The Timed "Up \& Go": a test of basic functional mobility for frail elderly persons. J Am Geriatr Soc 1991, 39(2):142-148

9. Rose DJ, Jones CJ, Lucchese N: Predicting the probability of falls in community-residing older adults using the 8-foot up-and-go: a new measure of functional mobility. JAPA 2002, 10(4):466-475.

10. Thomas Jl, Lane JV: A pilot study to explore the predictive validity of 4 measures of falls risk in frail elderly patients. Arch Phys Med Rehabil 2005, 86(8):1636-1640.

11. Arnold CM, Faulkner RA: The history of falls and the association of the Timed Up and Go test to falls and near-falls in older adults with hip osteoarthritis. BMC Geriatr 2007, 7:17.

12. Panel on Prevention of Falls in Older Persons. American Geriatrics Society and British Geriatrics Society: Summary of the Updated American Geriatrics Society/British Geriatrics Society clinical practice guideline for prevention of falls in older persons. J Am Geriatr Soc 2011, 59(1):148-157.

13. NICE: The assessment and prevention of falls in older people. 2013. http://www.nice.org.uk/CG161.

14. Rydwik E, Bergland A, Forséén L, Fräändin K: Psychometric properties of Timed Up and Go in elderly people: a systematic review. Physical \& Occupational Therapy in Geriatrics 2011, 29(2):102-25.
15. Beauchet O, Fantino B, Allali G, Muir SW, Montero-Odasso M, Annweiler C: Timed Up and Go test and risk of falls in older adults: a systematic review. J Nutr Health Aging 2011, 15(10):933-8.

16. Schoene D, Wu SM, Mikolaizak AS, Menant JC, Smith ST, Delbaere K, et al: Discriminative ability and predictive validity of the timed up and go test in identifying older people who fall: systematic review and meta-analysis. J Am Geriatr Soc 2013, 61(2):202-8.

17. Leeflang MM, Deeks JJ, Gatsonis C, Bossuyt PM: Systematic reviews of diagnostic test accuracy. Ann Intern Med 2008, 149(12):889-97.

18. Whiting P, Rutjes AW, Westwood ME, Mallett S, Deeks JJ, Reitsma JB, Leeflang MMG, et al: QUADAS-2: a revised tool for the quality assessment of diagnostic accuracy studies. Ann Intern Med 2011, 155(8):529-36.

19. Cousins G, Hijazze S, Van de Laar FA, Fahey T: Diagnostic accuracy of the ID Migraine: a systematic review and meta-analysis. Headache 2011, 51(7):1140-8.

20. Reitsma JB, Glas AS, Rutjes AW, Scholten RJ, Bossuyt PM, Zwinderman AH: Bivariate analysis of sensitivity and specificity produces informative summary measures in diagnostic reviews. J Clin Epidemio/ 2005, 58(10):982-90.

21. Swets JA: Measuring the accuracy of diagnostic systems. Science 1988 , 240(4857):1285-93.

22. Herman T, Mirelman A, Giladi N, Schweiger A, Hausdorff J: Executive control deficits as a prodrome to falls in healthy older adults: a prospective study linking thinking, walking, and falling. J Gerontol A Biol Sci Med Sci 2010, 65(10):1086-92.

23. Herman T, Giladi N, Hausdorff JM: Properties of the 'Timed Up and Go' test: more than meets the eye. Gerontology 2011, 57(3):203-10.

24. Beauchet O, Dubost V, Allali G, Gonthier R, Hermann FR, Kressig RW: 'Faster counting while walking' as a predictor of falls in older adults. Age Ageing 2007, 36(4):418-423.

25. Beauchet $\mathrm{O}$, Allali G, Annweiler C, Berrut G, Maarouf N, Herrmann FR, et al: Does change in gait while counting backward predict the occurrence of a first fall in older adults? Gerontology 2008, 54(4):217-23.

26. Sai AJ, Gallagher JC, Smith LM, Logsdon S: Fall predictors in the community dwelling elderly: a cross sectional and prospective cohort study. J Musculoskelet Neuronal Interact 2010, 10(2):142-50.

27. Trueblood PR, Hodson-Chennault N, McCubbin A, Youngclarke: Performance and impairment-based assessments among community dwelling elderly: sensitivity and specificity. J Geriatr Phys Ther 2001, 24(1):2-6.

28. Wrisley DM, Kumar NA: Functional gait assessment: concurrent, discriminative, and predictive validity in community-dwelling older adults. Phys Ther 2010, 90(5):761-73.

29. Pai Y-C, Wang E, Espy DD, Bhatt: Adaptability to perturbation as a predictor of future falls: a preliminary prospective study. J Geriatr Phys Ther 2010, 33(2):50-5.

30. Viccaro LJ, Perera S, Studenski SA: Is Timed Up and Go better than gait speed in predicting health, function, and falls in older adults? J Am Geriatr Soc 2011, 59(5):887-92.

31. Killough J: Validation of the Timed Up and Go Test to predict falls. J Geriatr Phys Ther 2006, 29(3):128-9.

32. Garber CE, Greaney ML, Riebe D, Nigg CR, Burbank PA, Clark PG: Physical and mental health-related correlates of physical function in community dwelling older adults: a cross sectional study. BMC Geriatr 2010, 10:6.

33. Okumiya K, Matsubayashi $K$, Nakamura T, Fujisawa M, Osaki $Y$, Doi $Y$, et al: The Timed "Up \& Go" test is a useful predictor of falls in community-dwelling older people. J Am Geriatr Soc 1998, 46(7):928-30.

34. Aoyama M, Suzuki Y, Onishi J, Kuzuya M: Physical and functional factors in activities of daily living that predict falls in community-dwelling older women. Geriatr Gerontol Int 2011, 11(3):348-57.

35. Yamada M, Uemura K, Mori S, Nagai K, Uehara T, Arai H, et al: Faster decline of physical performance in older adults with higher levels of baseline locomotive function. Geriatr Gerontol Int 2012, 12(2):238-46.

36. Yamada $M$, Ichihashi $N$ : Predicting the probability of falls in community-dwelling elderly individuals using the trail-walking test. Environ Health Prev Med 2010, 15(6):386-91.

37. Shimada H, Suzukawa M, Tiedemann A, Kobayashi K, Yoshida H, Suzuki T: Which neuromuscular or cognitive test is the optimal screening tool to predict falls in frail community-dwelling older people? Gerontology 2009, 55(5):532-8.

38. Melzer I, Kurz I, Shahar D, Oddsson L: Do voluntary step reactions in dual task conditions have an added value over single task for fall prediction? A prospective study. Aging Clin Exp Res 2010, 22(5-6):360-6.

39. Buatois S, Gueguen R, Gauchard GC, Benetos A, Perrin P: Posturography and risk of recurrent falls in healthy non-institutionalized persons aged over 65. Gerontology 2006, 52(6):345-52. 
40. Buatois S, Perret-Guillaume C, Gueguen R, Miget P, Vancon G, Perrin P, et al: A simple clinical scale to stratify risk of recurrent falls in community-dwelling adults aged 65 years and older. Phys Ther 2010, 90(4):550-60.

41. Lin MR, Hwang HF, Hu MH, Wu HD, Wang YW, Huang F: Psychometric comparisons of the timed up and go, one-leg stand, functional reach, and Tinetti balance measures in community-dwelling older people. $J$ Am Geriatr Soc 2004, 52(8):1343-8.

42. Russell MA, Hill KD, Blackberry I, Day LM, Dharmage SC: The reliability and predictive accuracy of the Falls Risk for Older People in the Community assessment (FROP-Com) tool. Age Ageing 2008, 37(6):634-9.

43. Thomas Jl, Lane L: A pilot study to explore the predictive validity of 4 measures of falls risk in frail elderly patients. Arch Phys Med Rehabil, 86(8):1636-1640.

44. Alexandre TS, Meira DM, Rico NC, Mizuta SK: Accuracy of Timed Up and Go Test for screening risk of falls among community-dwelling elderly. Rev Bras Fisioter 2012, 16(5):381-8.

45. Greene BR, Doheny EP, Walsh C, Cunningham C, Crosby L, Kenny RA: Evaluation of falls risk in community-dwelling older adults using body-worn sensors. Gerontology 2012, 58(5):472-80.

46. Bergland A, Jarnlo GB, Laake K: Predictors of falls in the elderly by location. Aging Clin Exp Res 2003, 15(1):43-50.

47. Shumway-Cook A, Brauer S, Woollacott M: Predicting the probability for falls in community-dwelling older adults using the Timed Up and Go test. Phys Ther 2000, 80(9):896-903.

48. Stenhagen M, Nordell E, Elmstahl S: Falls in elderly people: a multifactorial analysis of risk markers using data from the Swedish general population study 'Good Ageing in Skane'. Aging Clin Exp Res 2013, 25(1):59-67.

49. Rossat A, Fantino B, Nitenberg C, Annweiler C, Poujol L, Herrmann FR, et al: Risk factors for falling in community-dwelling older adults: which of them are associated with the recurrence of falls? J Nutr Health Aging 2010, 14(9):787-91.

50. Yamada M, Aoyama T, Nakamura M, Tanaka B, Nagai K, Tatematsu N, et al: The reliability and preliminary validity of game-based fall risk assessment in community-dwelling older adults. Geriatr Nurs 2011, 32(3):188-94.

51. Vaillant J, Martigné P, Vuillerme N, Caillat-Miousse JL, Parisot J, Juvin R, et al: Prediction of falls with performance on Timed "Up-and-Go" and one-leg-balance tests and additional cognitive tasks. Ann Réadapt Méd Phys 2006, 49(1):1-7.

52. Siggeirsdottir $\mathrm{K}$, Jonsson BY, Jonsson H Jr, Iwarsson S: The Timed 'Up \& Go' is dependent on chair type. Clin Rehabil 2002, 16(6):609-16.

53. Leveille SG, Jones RN, Kiely DK, Hausdorff JM, Shmerling RH, Guralnik JM, et al: Chronic musculoskeletal pain and the occurrence of falls in an older population. JAMA 2009, 302(20):2214-21.

54. Mackenzie L, Byles J, D'Este C: Validation of self-reported fall events in intervention studies. Clin Rehabil 2006, 20(4):331-9.

55. Buchner DM, Hornbrook MC, Kutner NG, Tinetti ME, Ory MG, Mulrow CD, Schechtman KB, et al: Development of the common data base for the FICSIT trials. J Am Geriatr Soc 1993, 41(3):297-308.

56. The prevention of falls in later life: A report of the Kellogg International Work Group on the Prevention of Falls by the Elderly. Dan Med Bull 1987, 34(4):1-24.

57. Anacker SL, Di Fabio RP: Influence of sensory inputs on standing balance in community-dwelling elders with a recent history of falling. Phys Ther 1992, 72(8):575-81.

58. Cumming RG, Sherrington C, Lord SR, Simpson JM, Vogler C, Cameron ID, Naganathan $V$, et al: Cluster randomised trial of a targeted multifactorial intervention to prevent falls among older people in hospital. BMJ 2008, 336(7647):758-760

59. Koski K, Luukinen $H$, Laippala $P$, Kivela S: Physiological factors and medications as predictors of injurious falls by elderly people: a prospective population-based study. Age Ageing 1996, 25(1):29-38.

\section{doi:10.1186/1471-2318-14-14}

Cite this article as: Barry et al:: Is the Timed Up and Go test a useful predictor of risk of falls in community dwelling older adults: a systematic review and meta- analysis. BMC Geriatrics 2014 14:14

\section{Submit your next manuscript to BioMed Central and take full advantage of:}

- Convenient online submission

- Thorough peer review

- No space constraints or color figure charges

- Immediate publication on acceptance

- Inclusion in PubMed, CAS, Scopus and Google Scholar

- Research which is freely available for redistribution

Submit your manuscript at www.biomedcentral.com/submit
C Biomed Central 